\title{
La tentative de légitimation du projet organisationnel européen par la communication de la commission européenne
}

Olivier Le Saëc

\section{QpenEdition}

\section{Journals}

Édition électronique

URL : http://journals.openedition.org/communicationorganisation/803

DOI : 10.4000/communicationorganisation.803

ISSN : $1775-3546$

Éditeur

Presses universitaires de Bordeaux

Édition imprimée

Date de publication : 1 juin 2009

Pagination : 158-169

ISSN : 1168-5549

Référence électronique

Olivier Le Saëc, « La tentative de légitimation du projet organisationnel européen par la communication de la commission européenne », Communication et organisation [En ligne], 35 | 2009, mis en ligne le 01 décembre 2012, consulté le 20 avril 2019. URL : http://journals.openedition.org/ communicationorganisation/803 ; DOI : 10.4000/communicationorganisation.803 


\section{La tentative de légitimation du projet organisationnel européen par la communication de la commission européenne}

Olivier Le Saëc

lesaecolivier@hotmail.com

Depuis cinquante ans, face à la nécessité pour les États européens d'organiser leurs relations autour des valeurs de paix et de développement économique afin d'établir les fondements d'une union sans cesse plus étroite entre les peuples, nous pouvons constater le développement d'une nouvelle forme organisationnelle publique, l'Union européenne. Cette nouvelle forme organisationnelle peut être appréhendée comme "une entité structurée de participants de différents pays qui coordonne leurs actions dans le but d'atteindre des objectifs communs » (Smouts, 1995: 12) se caractérisant par le fait que ce sont bien des «acteurs, relativement libres et autonomes, qui [ont créé] un système » (Bernoux, 1985 : 1290). Ainsi, les États européens coopèrent au sein d'une forme organisationnelle qui structure leurs conduites dans des relations d'interdépendance.

Nous pouvons alors constater qu'il s'est construit un système politique européen dont les "Traités de Rome et de Maastricht ont désigné la Commission européenne comme "l'exécutif» de l'Union européenne » (Smith, $2001: 3$ ), et, l'obligation de transposition des directives européennes en droit national permet à la Commission de revendiquer une forme de "domination légitime» sur la population européenne. Ainsi l'organisation publique supranationale, qu'est l'Union européenne, aurait les caractéristiques d' "une entreprise politique de caractère institutionnel dont la direction administrative revendique avec succès, dans l'application des règlements, [un certain] monopole de la contrainte physique légitime » (Weber, 1959 : 125).

Néanmoins, bien que l'Union européenne soit devenue une réalité, et que "la démocratie [soit] devenue le caractère politique commun de l'Europe de cette fin de siècle » (Morin, 1990 : 72), nous ne pouvons que constater la faiblesse du soutien populaire et l'émergence de critiques à l'encontre du projet organisationnel européen. Ainsi, alors que l'année 2005 devait être synonyme de la ratification du Traité établissant une Constitution pour l'Europe, nous n'avons pu que constater une forme de rejet de la construction et de l'intégration 
européenne par deux des pays fondateurs. Ces événements peuvent être interprétés comme un questionnement de la part des citoyens concernant la légitimité de l'Union, car «l'Union européenne subit de plein fouet la désaffection à l'égard du politique. [La] crise de la représentativité s'exprime encore plus à l'échelle européenne, la légitimité des institutions européennes n'apparaissant pas clairement aux yeux des citoyens » (Morin, 1990 : 245). Il est ainsi clair que la faiblesse de la participation des citoyens fait émerger des problématiques liées à la légitimité populaire du projet organisationnel européen, qui peuvent, à plus ou moins long terme, remettre en cause le projet organisationnel communautaire européen dans ses fondements.

Une des principales raisons présentées pour expliquer le manque de soutien au projet européen a été formulée comme résultant du manque d'information des citoyens, démontrant la conception que la communication a le pouvoir de renforcer les liens avec les citoyens car «l'extension de l'information a pour conséquence la participation de plus en plus grande du citoyen à la politique qui lui est appliquée » (Cotteret, 1997 : 113). Cette conception tend à conférer une place centrale aux activités de communication dans les sociétés démocratiques car il est désormais admis que «le système politique qui assure la régulation de l'ordre social dépend principalement de la fonction de communication» (Cotteret, 1997: 113), et, que «le développement de l'opinion fait partie de l'infrastructure démocratique de la Communauté et peut contribuer à la naissance, voulue par les traités européens, d'une "Europe organisée et vivante" »(Schuijt, 1972 : 20). Que nous révèle l'étude des pratiques de communication de l'Union européenne sur la légitimité de l'organisation publique européenne?

Nous nous intéresserons aux principes organisationnels de la communication de l'Union européenne qui nous permettront de prendre connaissance de l'institutionnalisation de cette dernière et de la responsabilité première de la Commission européenne. Ensuite, l'étude des pratiques de la politique de communication de l'Union nous permettra de prendre conscience de la marge de manœuvre limitée de l'exécutif européen pour contraindre les États membres d'appliquer la politique de communication déterminée collectivement. Et, dernièrement, nous réaliserons que la promotion et l'utilisation d'Internet dans la politique de communication de l'Union s'avère être un moyen afin de dépasser les leaders d'opinions, que sont les Etats membres, pour informer directement les citoyens et légitimer l'organisation supranationale. 
La tentative de légitimation du projet...

\section{Les principes organisationnels de la communication de la communication de l'Union européenne}

Dans cette première partie, nous allons prendre connaissance de la coopération interinstitutionnelle pour les activités de communication de l'Union, et, dans un second temps, nous verrons que la Commission s'avère avoir la responsabilité première pour la politique de communication de l'Union européenne.

La coopération interinstitutionnelle pour les activités de communication de l'Union européenne

En 2001, face au déclin constant de la participation des citoyens aux élections européennes, une tentative de réforme des activités de communication conduisit à l'émergence de principes organisationnels et à la clarification des responsabilités entre les Institutions européennes. Il en découle la création d'un nouveau cadre de coopération, le Groupe Interinstitutionnel de l'Information, qui concrétise l'émergence d'un leadership politique pour la stratégie de la communication. La grande avancée institutionnelle découlant de la réforme de la politique de communication de l'Union européenne est la création du Groupe Interinstitutionnel de l'Information (GII). Le GII est l'organe central de la coopération entre les Institutions européennes ayant comme fonction de «surveiller le bon fonctionnement de la coopération entre les institutions [et de] dresser le bilan de l'avancement et de définir des orientations au sujet des activités générales et spécifiques d'information" (Commission européenne, 2001: 8). Le GII est co-présidé par la Commission, le Parlement européen et le Conseil de l'Union européenne. Ainsi, dans le cadre du GII, les trois Institutions européennes sont représentées par des acteurs politiques, un vice-président pour le Parlement européen, la vice-présidente de la Commission, et le Ministre des Affaires européennes de l'État membre présidant le Conseil de l'Union européenne. Cette représentation au plus haut point hiérarchique et politique tend à dépasser les blocages techniques et structurels afin de donner des orientations politiques aux activités de communication de l'Union. Nous voyons que lors de la tentative de réforme de la politique de communication de l'Union, il fut pris en compte la nécessité de créer une structure regroupant les acteurs institutionnels à un niveau hiérarchiquement supérieur afin de définir les thèmes et les orientations de la politique de communication de l'Union. Ainsi, le Groupe Interinstitutionnel pour l'Information est devenu l'organe central de décision pour la politique de communication de l'Union, et, nous pouvons nous apercevoir que son leadership est renforcé par le 
rôle du Commissaire européen pour les relations institutionnelles et de la stratégie de communication qui essaye de donner un leadership politique sur la politique de communication de l'Union européenne. Donc, la création du Groupe Interinstitutionnel de l'Information est le symbole d'une prise de conscience par les autorités européennes de la nécessité d'une coopération interinstitutionnelle au plus au niveau hiérarchique possible afin de donner un leadership politique pour la politique de communication.

La responsabilité première de la Commission européenne pour les activités de communication communautaire

Au-delà des rôles que les Traités ont conférés à la Commission européenne, nous allons constater qu'elle a, en tant qu'exécutif du système politique européen, la responsabilité première pour les activités de communication. La Commission en tant qu'exécutif européen se retrouve en charge de l'organisation et de la réalisation de la politique de communication de l'Union européenne, et, il en découle que la Commission a la responsabilité première des activités de communication vis-à-vis des autres Institutions européennes. La Commission est la seule responsable des activités de communication et l'existence d'un problème de communication avec les citoyens a conduit à une prise de conscience, au point que la "Commission a fait de la communication l'un des objectifs stratégiques de son mandat, la reconnaissant pleinement comme une politique à part entière " (Commission européenne, $2005: 2$ ) se concrétisant par la création d'un poste de Commissaire européen en charge des relations interinstitutionnelles et de la stratégie de la communication.

\section{Les pratiques de la politique de communication de l'Union européenne}

Dans cette seconde partie, nous porterons notre attention sur les pratiques de la politique de communication de l'Union. Nous verrons que la Commission, du fait de sa responsabilité première pour les activités de communication, est l'organisatrice de la politique de communication de l'Union européenne. Néanmoins, nous constaterons son incapacité à contraindre les États membres d'appliquer la politique de communication.

\section{La Commission européenne, l'organisatrice de la communication}

La Commission a en charge d'élaborer les principaux messages de communication et de les tester via des «focus groups » dans chaque Etat membre avant de les proposer aux autres institutions européennes. Parallèlement, la Commission a en charge de 
" développer une stratégie de communication correspondant à chaque thème d'information prioritaire (messages, cibles, moyens d'action, calendrier, budget) »(Commission européenne, 2002 : 22). Et, «c'est sur cette base que la Commission proposera au Parlement européen et aux Etats membres de travailler ensemble pour la mise en œuvre des décisions prises au niveau du GII »(Commission européenne, 2002 : 22). Mais, même si la Commission et les autres Institutions définissent en commun les messages de communication et développent des stratégies de communication pour des thèmes d'information prioritaires, la mise en œuvre de la politique de communication repose sur la complémentarité de l'action des Institutions européennes et des Etats membres.

L'incapacité de la Commission de contraindre les Etats membres d'appliquer la politique de communication de l'Union européenne

Ainsi, bien que la Commission soit la responsable de la politique de communication de l'Union, il apparaît clairement que "sans le soutien actif des autorités nationales ou régionales les Institutions européennes demeureront dans l'incapacité d'atteindre ou de sensibiliser le citoyen lui-même » (Commission européenne, 2002: 21). Il s'avère que "Chaque Etat membre demeure libre de souscrire ou non à la stratégie ou aux messages proposés " (Commission européenne, 2002b: 22), bien qu'une fois la stratégie définie et validée, sa mise en œuvre repose sur la complémentarité de l'action des Institutions européennes et des Etats membres. Les documents de travail de l'Union confirment la «marge de manœuvre limitée » de la Commission en précisant que "les Etats membres ont réagi diversement aux avances de la Commission concernant les conventions; certains ont accueilli ces propositions avec enthousiasme et souhaiteront vraisemblablement développer et exploiter l'idée. D'autres ont accepté de participer dans le cadre d'objectifs étroitement circonscrits (par exemple, la campagne d'information sur l'euro) et limités dans le temps; il se peut qu'ils acceptent d'envisager d'autres questions telles que l'élargissement. Seuls deux États membres n'ont encore accepté aucune convention » (Commission européenne, 2001 : 13).

Ainsi, nous voyons clairement apparaître l'importance du rôle des Etats membres dans la politique de communication communautaire, mais, nous voyons aussi confirmer que " le contrôle de l'information est l'un des attributs du leader d'opinion pour Lewin qui utilise à son propos le nom de gatekeeper (en français, gardien, contrôleur ou sélectionneur de l'information) » (Maigret, 2003 : 78). Alors, force est 
de constater que même si "la Commission souhaite [délaisser] une approche allant du sommet vers la base, [et que] celle-ci placera le public au cour de son action et sera axée sur des thèmes intéressant les citoyens » (Commission européenne, mars 2005 : 4), il s'avère que la Commission est dépendante des Etats membres pour l'application de la politique de communication de l'Union dont elle est responsable, car, "le flux de communication n'est pas unidirectionnel et direct, pointé sur le seul récepteur, il est en réalité à deux étages et indirect, transitant par "le médiateur" » (Maigret, 2003 : 77), et que " sans le soutien actif des autorités nationales ou régionales les Institutions européennes demeureront dans l'incapacité d'atteindre ou de sensibiliser le citoyen lui-même» (Commission européenne, 2002: 21).

Ces phénomènes de responsabilité collective et de " non-réalisation » des activités de communication par les Etats membres mettent en évidence la liberté et l'autonomie de ces derniers dans le système de communication de l'Union européenne. Ainsi, nous pouvons conclure que les Etats membres «engagent leur autonomie dans les zones mal réglementées de l'organisation »(Bernoux, 1985: 132), et que le système de communication communautaire, comme dans tout construit humain, laisse une liberté relative aux Etats membres puisqu'ils apparaissent comme des gate keepers dont "le contrôle de l'information est l'un des attributs » (Maigret, 2003 : 78).

\section{La promotion et l'utilisation d'Internet dans la politique de communication de l'Union européenne}

Dans cette dernière partie, nous allons porter notre attention sur la promotion et l'utilisation d'Internet dans la politique de communication de l'Union européenne. Nous prendrons connaissance de la conception d'Internet par les Institutions européennes, et ensuite, nous porterons notre attention sur Europa, le portail Internet de l'Union européenne, qui nous permettra de concevoir l'utilisation d'Internet comme une tentative de dépassement des Etats membres.

\section{La conception d'Internet par les Institutions européennes}

Les Institutions européennes mettent en avant la promotion de la société de l'information dénommée «e-Europe 》 (Commission européenne, 2002a : 4) dans laquelle Internet aurait une place centrale dans la société future grâce aux programmes «e-Learning », «eGouvernement », « e-Health » et «e-Business » afin de réaliser «une nouvelle société basée sur la connaissance [devant] être ouverte à tous »(Commission européenne, 2002a : 4). 
$\mathrm{Au}$ travers de ses documents de travail, l'Union européenne précise que «le monde entier reconnaît que la société est globalement en train de changer - et continuera de se transformer - sous l'effet de l'évolution rapide des technologies de l'information et des communications, ce qui entraîne des changements économiques sociaux et culturels d'une ampleur jusqu'ici impensable " (Commission européenne, 2002a: 4). Les Institutions européennes précisent même que cette "évolution foudroyante des technologies de l'information et de la communication sera synonyme, pour l'Union européenne, d'une mutation économique et sociale si profonde qu'on peut déjà parler d'une troisième révolution industrielle » (Dacheux, 2004 : 115) car "le potentiel de l'Internet est quasiment illimité » (Commission européenne, 2007: 3). Cette «troisième révolution industrielle » n'introduira pas uniquement du changement d'ordre technologique, car ce changement « est profond et planétaire et il implique de nouvelles manières de communiquer, de nouvelles structures économiques et sociales et de nouveaux modes de gouvernance » (Commission européenne, 2002a : 4).

Ainsi, les Institutions européennes conceptualisent l'utilisation de l'Internet comme l'introduction d'un changement si profond qu'il conduira inéluctablement à l'avènement de la société de l'information. Il découle de ce projet de société "décloisonnée et transparente », la conception qu'Internet serait l'outil essentiel pour que «les pouvoirs publics commencent à s'engager dans une communication réellement réciproque sur l'Internet, qui devient l'expression d'une forme plus directe de la démocratie »(Commission européenne, 2007: 4), car, "l'Internet peut être le moteur de la transition d'une bureaucratie pesante vers une démarche véritablement tournée vers le citoyen» (Commission européenne, $2007: 5$ ).

\section{Europa, le portail Internet de l'Union européenne}

Suite à la réunion ministérielle du G7 sur la société de l'information organisée par la Commission à Bruxelles en février 1995, L'Union européenne a créé le site Internet Europa qui est rapidement devenu le portail Internet de l'Union européenne et de ses Institutions. Son but initial était de devenir un instrument s'inscrivant dans la politique de transparence de l'Union. Ce site Internet regroupe les informations diffusées par l'ensemble des Institutions de l'Union européenne. Europa a comme caractéristique de regrouper plusieurs millions de documents et plusieurs bases de données ouvertes au public avec un accès gratuit, dont, "entre novembre 2000 et novembre 2001, environ 500 millions de documents ont été consultés ou téléchargés depuis ce 
site» (Commission européenne, 2000c : 21), et, ses «utilisateurs principaux ont été le monde des affaires, les administrations nationales, les universités, les organisations non gouvernementales et les médias »(Commission européenne, 2000c : 21).

En 2001, la Commission ayant en charge la gestion d'Europa a constaté que "les possibilités d'interactivité qu'offre Internet restent peu exploitées [...et] tel qu'il se présente actuellement, le site de la Commission risque fort d'éclater en un labyrinthe de sites dispersés » (Commission européenne, 2000 : 7). Alors, en raison de la valeur stratégique que d'Europa pour l'avènement de la «e-Europe», la Commission a défini un plan de marche pour la réalisation d' « Europa deuxième génération "), visant à la mise en place de sites Web de la $2^{\text {ème }}$ génération fournissant la qualité de service attendue d'administrations modernes à l'époque de l'administration en ligne. Ainsi, pour les Institutions communautaires, «la Commission doit être dans le peloton de tête en ce qui concerne la mise en ouvre des techniques faisant appel à l'Internet, car il n'y a pas d'autre voie de salut pour faire face à l'accroissement de la demande et pour offrir un service vraiment digne de ce nom » (Commission européenne, 2001 : 9). Ainsi, nous voyons que la Commission souhaite développer la société de l'information en plaçant "l'Internet au centre des activités de communication dans le contexte du programme "Commission en ligne" " (Commission européenne, 2007: 3). Mais, en plus de développer Europa afin de promouvoir la société de l'information, l'utilisation d'Internet par la Commission peut être appréhendée comme une tentative de dépassement des Etats membres pour informer directement les citoyens.

En effet, alors que le fonctionnement du système de communication européen confère une place centrale aux Etats membres et que la Commission n'a pas le pouvoir de les contraindre d'appliquer la politique de communication sur l'Union, l'utilisation d'Internet apparait être une tentative de contournement de ces gate keepers en valorisant directement son action auprès des citoyens afin d'obtenir une légitimité populaire. Et, à l'heure actuelle, «le principal enjeu pour la Commission européenne dans son ensemble est de garantir que ses activités et ses communications liées à l'Internet reposent sur un contenu attrayant et une technologie de pointe» (Commission européenne, 2007: 14) afin de structurer "une communication réellement réciproque sur l'Internet, qui devient l'expression d'une forme plus directe de la démocratie » (Commission européenne, 2007: 4). Néanmoins, la Commission européenne reconnaît qu'à l'heure actuelle «Europa reste encore méconnu de la majorité des 
citoyens de l'Union » (Commission européenne, 2007 : 6). Donc, nous voyons que la dépendance de la Commission vis-à-vis des Etats membres pour l'application de la politique de communication tend à appréhender l'utilisation d'Internet comme une tentative de dépassement des États membres pour communiquer directement avec les citoyens. La Commission précise même que "l'Internet semble jouer un rôle de plus en plus important en tant que moyen rentable de communication avec les citoyens d'Europe et au-delà, notamment avec des groupes cibles spécifiques» (Commission européenne, $2007: 17)$.

\section{Conclusion}

Le transfert progressif de compétences des Etats membres vers l'Union européenne a conduit au développement et à l'institutionnalisation des activités de communication, car, l'évolution des Communautés européennes en Union européenne signifie la réalisation d'une Union politique et la nécessité d'obtenir un support populaire, une légitimité populaire nécessaire à tout système politique démocratique. L'Union européenne est avant tout un construit organisationnel, un consensus entre des acteurs ne répondant pas seulement à des objectifs communs, mais surtout à des logiques individuelles d'acteurs. Donc, nous conclurons que la Commission, en tant que responsable de la politique de communication de l'Union européenne, tente d'utiliser un outil de communication afin de légitimer l'existence de l'Union européenne auprès des citoyens "pour résoudre, le mieux possible, un problème lié à une situation de [son] existence » (Mucchielli, 2000: 193). Néanmoins, bien que l'utilisation d'Internet s'avère être une tentative de dépassement des Etats membres afin de structurer autrement le système de communications communautaire, il demeure que nous ne pouvons que constater que la légitimité de l'Union européenne n'a que comme fondement "le pouvoir tel que l'exerce le "serviteur de l'Etat" » (Weber, 1959 : 127) qu'est la Commission européenne.

\section{Bibliographie}

BERNOUX P., La sociologie des organisations, Editions du Seuil. Paris.1985.

COMMMISSION EUROPEENNE, Communiquer sur L'Europe par l'Internet. Faire participer les citoyens. SEC (2007)1742. Bruxelles, le 21 décembre 2007. 
Dossier : Repenser la communication des organisations publiques

COMMMISSION EUROPEENNE, Plan d'action de la Commission relatif à l'amélioration de la communication sur l'Europe. Communication à la Commission européenne. 20 juillet 2005.

COMMISSION EUROPEENNE, Stratégie politique annuelle pour 2006. Communication de la commission au Parlement européen et au Conseil. Mars 2005.

COMMISSION EUROPEENNE, L'Europe en Mouvement, vers une Europe de la connaissance. Communication de la Commission européenne. Octobre 2002.

COMMMISSION EUROPEENNE, Une stratégie d'information et de communication pour l'Union européenne - Communication de la Commission européenne. COM (2002) 350 final. Le 2.7.2002.

COMMMISSION EUROPEENNE, Vers la Commission en ligne : Europa 2éme génération. Communication de la commission européenne. Service presse et communication. Bruxelles. 07.2001. D (2000).

COMMMISSION EUROPEENNE, Un nouveau cadre de coopération pour les activités concernant la politique d'information et de communication de l'Union européenne. Communication de la Commission européenne. COM (2001) 354 final. 27.6.2001.

COMMMISSION EUROPEENNE, Livre blanc sur la réforme. Document de la commission européenne. $\operatorname{COM}(2001)$. 428 final. Bruxelles. 2001

COMMMISSION EUROPEENNE, Rapport général de l'Union européenne de 2001.

COTTERET J. M. Gouverner, c'est paraître. Quadrige/P.U.F. 1997.

DACHEUX E., Ne pas confondre le symptôme et la maladie. Analyse critique de la politique de communication de l'Union européenne. XIV congrès national de la SFSIC. Béziers. 2004.

MAIGRET E., Sociologie de la communication et des médias. Armand Colin. 2003.

MORIN E., Penser l'Europe. Paris. Editions Gallimard. Paris. 1990.

MORIN E., Les sept savoirs nécessaires à l'éducation du futur. Seuil. Paris. 1999.

MUCCHIELLI A., La nouvelle communication. Armand Colin. Paris. 2000. 
La tentative de légitimation du projet...

SCHUIJT. Rapport sur la politique d'information des Communautés européennes. Parlement européen. 7 février 1972.

SMITH A., Qu'est-ce que la Commission européenne?, in, La Commission européenne en politique(s). Revue Pôle Sud N 15. 2001.

SMOUTS M.-C., Les organisations internationales. Armand Colin. 1995.

WEBER M., Le savant et le politique. Paris. Plon. 1959.

ZORGBIBE C., Les relations internationales. Presses Universitaires de France. Collection Thémis. $5^{\text {ème }}$ édition. 1994. 